\title{
The Functional Link Between AMPK Pathway Activation and Atherosclerosis Progression
}

\author{
Kholoud Alaa El Mihi* \\ Department of Biochemistry, Faculty of Pharmacy, Mansoura University, Egypt
}

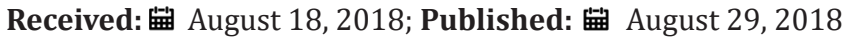

*Corresponding author: Kholoud Alaa El Mihi, Department of Biochemistry, Faculty of Pharmacy, Mansoura University, Mansoura, Egypt

\begin{abstract}
The atherosclerosis is a complex pathological process that influences lipid deposition in the intima of middle- and large-sized arteries. AMPactivated protein kinase (AMPK) is a nutrient sensor that stimulates ATP production and fatty acid oxidation, but inhibits lipogenesis. Moreover, AMPK plays a key role in regulating oxidative stress, inflammation and vascular smooth muscle cells de-differentiation involved in atherosclerosis progression.
\end{abstract}

Keywords: Atherosclerosis; Oxidative stress; AMPK; Dyslipidemia

Abbreviations: ACC: Acetyl COA Carboxylase; AMPK: AMP-Activated Protein kinase; ECM: Extracellular Matrix; LDL: Low-Density Lipoprotein; NO: Nitric Oxide; NOX: NADPH Oxidase; VSMCs: Vascular Smooth Muscle Cells

\section{Introduction}

\section{Atherosclerosis}

Atherosclerosis is considered as one of causes of morbidity and premature disability in developed countries. Atherosclerosis is a complex chronic pathological disorder that affects many organs in human body causing ischemia of brain, extremities or heart leading to infarction depending on vessel involved [1]. It is characterized by the subendothelial accumulation of fibrofatty deposits in the intima of large arteries. Atherosclerosis was formerly regarded as a bland lipid storage disease, but recent studies have illustrated the role of inflammation, oxidative stress, immune system and endothelium on progression of atherosclerotic lesions [2]. Major risk factors for atherosclerosis involve hypercholesterolemia, dyslipidemia, hypertension, diabetes, inflammation, smoking, obesity, old age and family history of heart diseases [3].

\section{Biochemical mechanisms of Atherosclerosis}

Atherosclerosis is a progressive process that adversely influences the endothelial-lining of arteries. Additionally, vascular endothelium is considered as a functional barrier between blood stream and arterial wall. Vascular endothelium maintains the vascular homeostasis through secreting of contracting factors (e.g. endothelin and angiotensin II) and relaxing factors [e.g. nitric oxide (NO)] [4]. Imbalance of endothelial functions resulting in injury is called endothelial dysfunction, the initial stage of atherosclerosis. The process of atherosclerosis can be summarized as follow $[2,3,5]$. a) Injured vascular endothelial cells release chemokines and cytokines and also express adhesion molecules.

b) Monocytes are chemoattracted to the injured endothelium and penetrate into the subendothelial spaces where they differentiate into macrophages.

c) High blood levels of low-density lipoprotein (LDL) cholesterol infiltrates into subendothelial spaces where it is oxidized, modified and taken up by activated macrophages leading to foam cell formation.

d) Vascular smooth muscle cells (VSMCs) migrate into the intima, transform and proliferate into extracellular matrix (ECM) secreting cells. Moreover, oxidized LDL are taken up by transformed VSMCs gaining them atherogenic properties.

e) The increase in ECM secretion by proliferative VSMCs leading to the formation of fibrous scar and atherosclerosis.

f) The vital risks of atherosclerosis are that atherosclerotic plaques may block the vessels and obstruct blood flow, or they may rupture under the influence of biological and mechanical forces leading to thrombosis and clot formation [6].

\section{AMPK Pathway}

AMP-activated protein kinase (AMPK) is a metabolic key that maintains the balance of ATP production under many physiological 
conditions. AMPK is present as a heterotrimeric complex containing $\alpha$-catalytic subunit with Ser/Thr kinase, $\beta$-regulatory subunit with a carbohydrate binding domain and $\gamma$-subunit with cystathionine$\beta$-synthase domains [7]. The downstream effectors of AMPK affect many vital cellular processes involving lipid metabolism [Hydroxymethyl glutaryl CoA reductase; Acetyl CoA carboxylase (ACC)], carbohydrate metabolism (6-phosphofructo-2-kinase; glycogen synthase), ion transport [cystic fibrosis transmembrane conductance regulator (CFTR)] and cell signaling (endothelial nitric oxide synthase; insulin receptor substrate-1) [8].

\section{AMPK Activation and Dyslipidemia}

Dyslipidemia is defined as elevated LDL cholesterol and triglyceride and/or decreased high density lipoprotein cholesterol and it is a primary factor inducing endothelial dysfunction [9]. The serine kinase AMPK plays a major role in lipid metabolism. In addition, AMPK mitigates lipogenesis and elevates fatty oxidation by affecting downstream signaling as ACC [10]. ACC has been regarded a key target in the treatment of metabolic diseases as type 2 diabetes and dyslipidemia owing to its role in lipogenesis [11]. Activated AMPK inhibits ACC activity through phosphorylation leading to a decline in malonyl CoA content and an increase in $\beta$-oxidation [12].

\section{AMPK Activation and Oxidative Stress}

Oxidative stress is characterized by the increase in reactive oxygen species and free radicals. Reactive oxygen species involve oxides and hydroperoxides, these compounds are produced during the oxidation-reduction reactions [13]. Recent studies have shown that AMPK has anti-oxidant activities that are partially attributed to the inhibition of NADPH oxidase (NOX) isoforms (NOX2; NOX4). As NOX is the enzyme responsible for reactive oxygen species generation [14]. In addition, AMPK activation regulates mitochondrial biogenesis and antioxidant defense gene expression. Furthermore, the expression levels of $\gamma$-glutamyl cysteine synthetase, catalase and superoxide dismutase, critical antioxidant enzymes, were downregulated after knockdown of the AMPK $\alpha 1$ subunit [15].

\section{AMPK Activation and Macrophages}

Circulating monocytes have a vital role in atherosclerosis formation and progression by influencing the plaque structure and the inflammatory response. AMPK activation reduces monocytes chemoattraction by suppressing chemokine production. Additionally, AMPK activation attenuates the number of macrophages in the atherosclerotic plaques [16]. Moreover, the phosphorylated AMPK suppresses the pro-inflammatory responses, macrophage polarization and inflammatory cytokine production [17]. Another study showed that activation of AMPK by phosphorylation plays anti-inflammatory activities through the AMPK/Nrf2 signaling pathways and its downstream target genes, (Hemoxygenase-1 and NADPH dehydrogenase quinone-1), in the macrophages, so preventing excessive macrophages-related responses in inflammation [18].

\section{AMPK Activation and VSMCs}

In healthy individuals, the arterial wall contains contractiontype VSMCs long-spindle shaped in the middle layer of intima. Although, in response to multiple stimuli, VSMCs dedifferentiate into proliferative and ECM-secreting cells [19]. Many studies have shown that AMPK can regulate cell differentiation through many downstream signaling pathways including endothelial nitric oxide synthase [20] and also promote re-differentiation of VSMCs [21]. Additionally, proliferative VSMCs enter S phase of the cell cycle, unlike the normal to be arrested in the G0/G1 phase of the cell cycle. AMPK activation was found to induce cell cycle arrest of VSMCs through p53 and p21 downstream [22].

\section{References}

1. Wang T, Butany J (2017) Pathogenesis of atherosclerosis. Diagn Histopathol 23(11): 473-478.

2. Rao PS, Kiranmayi V (2012) Biochemical mechanisms underlying atherogenesis. J Clin Sci Res 1: 24-34.

3. Rafieian Kopaei M, Setorki M, Doudi M, Baradaran A, Nasri H (2014) Atherosclerosis: Process, Indicators, Risk Factors and New Hopes. Int J Prev Med 5(8): 927-946.

4. Zmyslowski A, Szterk A (2017) Current knowledge on the mechanism of atherosclerosis and pro-atherosclerotic properties of oxysterols. Lipids Health Dis 16(1): 188.

5. Katakami N (2018) Mechanism of Development of Atherosclerosis and Cardiovascular Disease in Diabetes Mellitus. J Atheroscler Thromb 25(1): 27-39.

6. Kwak BR, Back M, Bochaton Piallat ML, Caligiuri G, Daemen MJ, et al. (2014) Biomechanical factors in atherosclerosis: mechanisms and clinical implications. Eur Heart J 35 (43): 3013-3020, 3020a-3020d.

7. Hardie DG, Schaffer BE, Brunet A (2016) AMPK: An Energy-Sensing Pathway with Multiple Inputs and Outputs. Trends Cell Biol 26(3): 190201.

8. Shirwany NA, Zou MH (2010) AMPK in cardiovascular health and disease. Acta Pharmacol Sin 31(9): 1075-1084.

9. Haznedaroglu IC, Malkan UY (2017) Lipotoxicity-Related Hematological Disorders in Obesity. Adv Exp Med Biol 960: 469-487.

10. Hardie DG (2014) AMP-activated protein kinase: a key regulator of energy balance with many roles in human disease. J Intern Med 276(6): 543-559.

11. Bourbeau MP, Bartberger MD (2015) Recent Advances in the Development of Acetyl-CoA Carboxylase (ACC) Inhibitors for the Treatment of Metabolic Disease. J Med Chem 58(2): 525-536.

12. Ong KW, Hsu A, Tan BKH (2013) Anti-diabetic and anti-lipidemic effects of chlorogenic acid are mediated by ampk activation. Biochem Pharmacol 85(9): 1341-1351.

13. Dong GZ, Lee JH, Ki SH, Yang JH, Cho IJ, et al. (2014) AMPK activation by isorhamnetin protects hepatocytes against oxidative stress and mitochondrial dysfunction. Eur J Pharmacol 740: 634-640.

14. Chen Y, Chen C, Dong B, Xing F, Huang H, et al. (2017) AMPK attenuates ventricular remodeling and dysfunction following aortic banding in mice via the Sirt3/Oxidative stress pathway. Eur J Pharmacol 814: 335-342.

15. Colombo SL, Moncada S (2009) AMPKalpha1 regulates the antioxidant status of vascular endothelial cells. Biochem J 421(2):163-169.

16. Wang J, Ma A, Zhao M, Zhu H (2017) AMPKactivation reduces the number of atheromata macrophages in ApoE deficient mice. Atherosclerosis 258: 97-107. 
17. Xiong XQ Geng Z, Zhou B, Zhang F, Han Y, et al. (2018) FNDC5 attenuates adipose tissue inflammation and insulin resistance via AMPK-mediated macrophage polarization in obesity. Metabolism 83: 31-41.

18. Park SY, Jin ML, Yi EH, Kim Y, Park G (2018) Neochlorogenic acid inhibits against LPS-activated inflammatory responses through up-regulation of Nrf2/HO-1 and involving AMPK pathway. Environ Toxicol Pharmacol 62: $1-10$.

19. Gomez D, Owens GK (2012) Smooth muscle cell phenotypic switching in atherosclerosis. Cardiovasc Res 95(2): 156-164.

\section{ISSN: 2574-1241}

DOI: 10.26717/BJSTR.2018.08.001664

Kholoud Alaa El Mihi. Biomed J Sci \& Tech Res

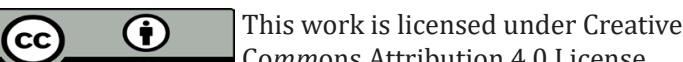

Submission Link: https://biomedres.us/submit-manuscript.php
20. Li X, Han Y, Pang W, Li C, Xie X, et al. (2008) AMP-activated protein kinase promotes the differentiation of endothelial progenitor cells. Arterioscler Thromb Vasc Biol 28 (10): 1789-1795.

21. Liu Z, Zhang M, Zhou T, Shen Q, Qin X (2018) Exendin-4 promotes the vascular smooth muscle cell re-differentiation through AMPK/SIRT1/ FOX03a signaling pathways. Atherosclerosis 276: 58-66.

22. Chan KC, Huang HP, Ho HH, Huang CN, Lin MC, et al. (2015) Mulberry polyphenols induce cell cycle arrest of vascular smooth muscle cells by inducing NO production and activating AMPK and p53. J Funct Foods 15: 604-613.

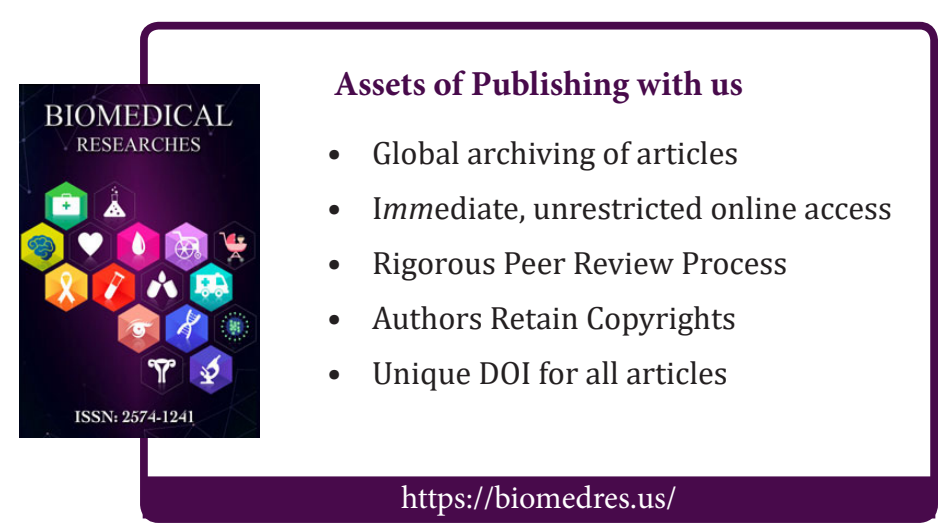

